Revue d'histoire de l'Amérique française

REVUE D.HISTOIRE DE L'AMÉRIQUE FRANÇAISE

\title{
Inventaire critique des notaires royaux des gouvernements de Québec, Montréal et Trois-Rivières (1663-1764) (suite)
}

\section{André Vachon}

Volume 10, numéro 2, septembre 1956

URI : https://id.erudit.org/iderudit/301764ar

DOI : https://doi.org/10.7202/301764ar

Aller au sommaire du numéro

Éditeur(s)

Institut d'histoire de l'Amérique française

ISSN

0035-2357 (imprimé)

1492-1383 (numérique)

Découvrir la revue

Citer cet article

Vachon, A. (1956). Inventaire critique des notaires royaux des gouvernements de Québec, Montréal et Trois-Rivières (1663-1764) (suite). Revue d'histoire de l'Amérique française, 10(2), 257-262. https://doi.org/10.7202/301764ar d'utilisation que vous pouvez consulter en ligne. 


\section{INVENTAIRE CRITIQUE DES NOTAIRES ROYAUX DES GOUVERNEMENTS DE QUÉBEC, MONTREAL * ET TROIS-RIVIËRES \\ (1663-1764)}

\section{CHAPITRE DEUXIEME \\ JEAN CUSSON}

Le 16 avril 1669, Bouteroue donnait une commission à Jean Cusson pour exercer les charges de procureur fiscal, notaire et greffier en la seigneurie du Cap de la Madeleine, précisant que Cusson «exercera la dite charge [notaire] en qualité de notaire royal et non autrement $» .^{9}$ M. J.-Edmond Roy a vu dans cette commission une manifestation de la volonté centralisatrice de l'intendant, au détriment des droits des seigneurs. ${ }^{10}$

Cusson pratiqua comme notaire dans la seigneurie de Champlain de 1687 à 1700, puis dans la région de Montréal jusqu'en 1704. ${ }^{11}$ Nous n'avons pas retrouvé de commission lui permettant d'instrumenter dans le gouvernement de Montréal. Cusson décéda à St-Sulpice, le 8 avril $1712 .{ }^{12}$

Son greffe est conservé partie aux Archives judiciaires des Trois-Rivières et partie aux Archives judiciaires de Montréal.

\section{PIERRe Petit}

Le 4 août 1721, Bégon «donnait et octroyait [...] led. office de $\mathrm{No}^{\text {re }}$ Royal en la jurisd. ${ }^{\text {on }}$ des Trois rivieres aud. $\mathrm{S}^{\mathrm{r}}$ Pierre Petit $\gg^{13}$

\footnotetext{
9 L'original de cette commission est à la bibliothèque municipale de Montréal. M. J.-E. Roy l'a citée dans Histoire du Notariat au Canada, I: 201 ; voir aussi P.-G. Roy, Ord. comm., I: 88.

10 J.-E. Roy, op. cit., I: 200 s.

$11 R A P Q, 1921-22$ : 25.

12 Ibid.

$13 \mathrm{APQ}$, Ord. Int., 71/2: 185.
} 93-103.

* Voir notre Revue, vol. IX, no $3: 423-438$; IX, no $4: 546-561$; X, no 1 : 
Petit fut également substitut du procureur du roi de la juridiction des Trois-Rivières, en vertu d'une commission en date du 2 septembre $1721 .{ }^{14}$

Le 18 novembre 1721, avait lieu l'information de vie et mœurs de Pierre Petit, bourgeois des Trois-Rivières, pourvu par l'intendant des charges de substitut du procureur du roi et de notaire royal en la juridiction des Trois-Rivières..$^{15}$

Petit décéda aux Trois-Rivières, le 24 avril $1737 .{ }^{16}$

Son greffe est conservé aux Archives judiciaires des TroisRivières.

\section{ARnould Balthazar Pollet}

Voir l'inventaire des notaires royaux du gouvernement de Québec, page 26.

\section{JOSEPH ROUILLARD}

Voir l'inventaire des notaires royaux du gouvernement de Québec, page 28.

\section{Hyacinthe-Olivier Pressé}

Le premier octobre 1735, Pierre Petit n'étant plus en état d'exercer, vu son grand âge et ses infirmités, l'intendant Hocquart commet le sieur Hyacinthe-Olivier Pressé pour « remplir et exercer lesd. offices de greffier delad Jurisdiction [TroisRivières], ensemble de Notaire Royal et d'huissier en Icelle ».17 Le 7 novembre, avait lieu l'information de vie et mœurs. ${ }^{18}$

En 1746, au cours d'une querelle, Pressé eut le malheur de tuer un certain Heu dit Millet. D'abord condamné à mort, Pressé vit sa sentence commuée en une condamnation aux galères à perpétuité.

Pressé avait cessé de pratiquer en 1643 , comme en fait foi la commission de Joseph Caron, son successeur. ${ }^{19}$

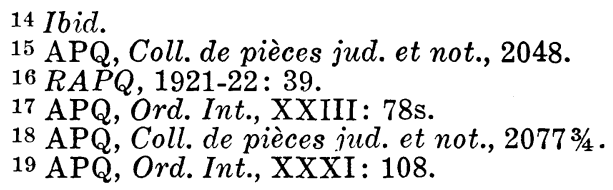


Le greffe de Pressé est conservé aux Archives judiciaires des Trois-Rivières.

\section{JOSEPH CARON}

Caron, né à Ste-Anne, en 1686, fut enseveli aux TroisRivières le 15 février $1746 .{ }^{20}$

Le 31 octobre 1743, Pressé ayant quitté sa charge, Hocquart commet Joseph Caron pour exercer l'office de greffier et de notaire royal en la juridiction des Trois-Rivières. ${ }^{21}$

Le greffe de Caron est aux Archives judiciaires des TroisRivières.

\section{LoUis Pillard}

Voir l'inventaire des notaires royaux du gouvernement de Québec, page 30.

\section{JEAN LEPROUST}

Le 27 juillet 1746, l'intendant nommait Jean Leproust notaire royal en la juridiction des Trois-Rivières et dans toute l'étendue du gouvernement de cette ville. ${ }^{22}$

On possède, daté du premier août 1746 , un jugement portant réception en l'office de notaire royal pour la juridiction des Trois-Rivières du Sieur Jean Leproust, praticien. ${ }^{23}$

Leproust, qui était originaire du diocèse de Poitiers, ${ }^{24}$ exerça jusqu'en 1761, alors qu'il s'embarqua pour la France. On ne saurait dire si Leproust avait reçu une nouvelle commission sous le régime militaire. Nous avons déjà dit qu'aucune commission de notaire n'a été retrouvée aux Trois-Rivières, pour la période de 1759 à 1764 .

Le greffe de Leproust est aux Archives judiciaires des Trois-Rivières.

20 Tanguay, op. cit., II : 550 .

21 APQ, Ord. Int., XXXI: 108.

22 APQ, Ord. Int., XXXIV: 63. Dans cette commission, Leproust est appelé «Prou 》.

$23 \mathrm{APQ}$, Coll. de pièces jud. et not., 2117.

24 Tanguay, op. cit., VI: 460. 


\section{Pierre-François Rigault}

Le 20 avril 1748, Bigot nommait Pierre-François Rigault, huissier au conseil supérieur de Québec, pour exercer un office de notaire royal dans l'étendue des côtes « et compris » la Pointedu-Lac, du côté du nord en remontant «jusques et compris » le chenail du nord, et «depuis et compris » la paroisse de Nicolet du côté du sud «jusques et compris » le chenail du Moine, dans le gouvernement des Trois-Rivières. ${ }^{25}$

Rigault, qui était fils de famille, arriva au pays comme soldat, en $1726 .{ }^{26}$ Il exerça jusqu'en 1778.

Son greffe est conservé aux Archives judiciaires des TroisRivières.

\section{Nicolas Duclos}

Le 7 juillet 1753, Bigot désigna Nicolas Duclos pour exercer l'office de notaire royal dans les côtes depuis et compris Batiscan jusques et compris le Cap-de-la-Madeleine. ${ }^{27}$

Le commissaire-ordonnateur Varin, le 21 décembre 1754, permet à Duclos de passer tous les actes de notoriété dont il pourra être requis par les habitants de la seigneurie de St-Pierreles-Becquets. ${ }^{28}$

Duclos pratiqua jusqu'en 1769 .

Son greffe est aux Archives judiciaires des Trois-Rivières.

Des trois gouvernements de la Nouvelle-France, c'est celui, pourtant moins considérable, des Trois-Rivières, qui offre le plus de difficulté au point de vue de l'étude du notariat. Tous les notaires de la première période, peut-être favorisés par l'éloignement de la Capitale, s'y disent notaires royaux; par contre, la commission de la plupart d'entre eux ne nous est pas parvenue. C'est ainsi que dans tout le gouvernement, on ne trouve, en un

$25 \mathrm{APQ}$, Ord. Int., XXXVI: 67.

$26 R A P Q, 1921-22: 51$.

27 APQ, Ord. Int., XL: 66.

28 Ibid., XLI: 2. Duclos est ici nommé Claude, par erreur. 
siècle, que dix notaires dont la qualité de notaires royaux soit absolument certaine. De Jean Cusson, le premier notaire royal du gouvernement des Trois-Rivières, à Pierre Petit, second notaire royal dont nous possédions la commission, il s'écoule plus d'un demi-siècle, pendant lequel plusieurs notaires exercèrent dans le gouvernement mais dont nous ne pouvons dire s'ils furent notaires royaux. Nous ferons maintenant l'inventaire de ces notaires dont la qualité reste forcément douteuse.

\section{SÉVÉRIN AMEAU}

On ne connaît aucune commission de notaire accordée à Séverin Ameau. Le premier acte que nous avons de lui est daté du 2 février 1651; le deuxième porte la date du 21 mai 1652. C'est à partir de 1652, semble-t-il, peu après son arrivée au pays, que le notaire Ameau commença à exercer régulièrement.

Ameau se disait notaire royal: il ne pouvait l'être avant 1663 , comme nous l'avons expliqué pour les notaires du gouvernement de Québec. A-t-il reçu par la suite une commission de notaire royal ? On ne saurait le dire.

Ameau naquit à St-Sauveur de Paris, en 1619, et fut enseveli aux Trois-Rivières le 9 mai 1715 , à l'âge de quatre-vingt-seize ans. ${ }^{29}$ Il avait pratiqué pendant cinquante ans.

Son greffe est aux Archives judiciaires des Trois-Rivières..$^{30}$

\section{Guillaume De La Rue}

Guillaume de La Rue, d'après l'Archiviste de la Province, « exerça comme notaire royal dans la seigneurie de Champlain de 1664 à $1689 \gg^{31}$ Nous n'avons cependant aucune commission qui puisse attester que La Rue était notaire royal. Il est vrai qu'il en prenait le titre, mais de cela nous ne pouvons rien conclure de certain. D'autre part, les textes officiels le disent simplement notaire. ${ }^{32}$

29 Tanguay, op. cit., I: 5.

30 Voir Invent. des greffes des not., XI : 49-164.

31 RAPQ, 1921-22: 22.

32 Jug. et délib., I: 796. 
Guillaume de La Rue fut également juge de la seigneurie de Champlain. Il était né à St-Maclou, en 1636, et il décéda à SteAnne-de-la-Pérade en janvier 1717. ${ }^{33}$

Ce qu'on a conservé des actes de La Rue est aux Archives judiciaires des Trois-Rivières.

André VACHON,

(à suivre)

33 Tanguay, op. cit., I: 351. 